\title{
Áreas de congregación de cetáceos en el Pacífico norte de Costa Rica: recomendaciones para la gestión del recurso
}

\author{
Damián Martínez-Fernández ${ }^{1}$, Andrea Montero-Cordero ${ }^{2} \&$ David Palacios-Alfaro $^{3}$ \\ 1. Proyecto Consolidación de Áreas Marinas Protegidas de Costa Rica SINAC-PNUD-GEF, San José, Costa Rica; \\ damian.martinez@sinac.go.cr \\ 2. Asociación Costa Rica Por Siempre, San José, Costa Rica; amontero@costaricaporsiempre.org \\ 3. Fundacion Keto, Apdo 1735-1002 San José, Costa Rica; dpalacios@fundacionketo.org
}

Recibido 02-X-2013. Corregido 11-VI-2014. Aceptado 27-IX-2014.

\begin{abstract}
Cetacean congregation areas in the North Pacific of Costa Rica: management recommendations. The information on cetacean distribution in the North Pacific of Costa Rica lacks a conservation-oriented analysis. The aim of this study is to identify areas of importance for cetaceans, especially outside the of the existing protected areas. Previously published data on cetacean sightings between 2004 and 2008 were analyzed, and supplemented by survey effort during the dry season of 2012. A Kriging geo-statistical analysis was used to predict the spatial continuity of cetacean sightings within four study areas. The most important congregation areas for dolphins are: Bahía Santa Elena, Parque Nacional Marino Las Baulas and Reserva Natural Cabo Blanco, they have high densities of dolphin feeding groups. Relevant congregation areas for humpback whales are: Bahía Santa Elena, Playa Tambor, Golfo de Papagayo and Reserva Natural Cabo Blanco. We found areas of high cetacean concentration in the North Pacific, both inside and outside protected areas. Some of the non-protected areas should be prioritized for conservation: Bahía Santa Elena, Bahía Culebra and the surrounding areas of Punta Pargos and Reserva Natural Cabo Blanco. Rev. Biol. Trop. 62 (Suppl. 4): 99-108. Epub 2014 Diciembre 01.
\end{abstract}

Key words: spotted dolphin, humpback whale, marine protected areas, management, conservation gaps.

En los últimos años en Costa Rica se han realizado valiosos esfuerzos de investigación y conservación de ambientes marinos. El primer inventario de especies marinas en el 2009 determinó que el país posee 6.778 especies marinas identificadas $(3,5 \%$ del total mundial) en tan solo $0,16 \%$ de la superficie total marina del planeta (Wehrtmann \& Cortés, 2009). Al mismo tiempo, por medio del Sistema Nacional de Áreas de Conservación (SINAC) se desarrolló un análisis de representatividad biológica y amenazas para la identificación de sitios de importancia para la conservación de ambientes marinos (SINAC, 2008). Estos se reconocieron como sitios prioritarios para la atención de la biodiversidad marina, que actualmente se conocen como "se conocen como sitios de importancia para la conservación, antiguamente conocidos como sitios de importancia para la conservación marina" (SINAC, 2008). Estos vacíos tienen el propósito de optimizar los esfuerzos nacionales de conservación in situ de la biodiversidad marina del país.

Para el Pacífico norte se determinaron cuatro sitios de importancia para la conservación priorizados a nivel nacional, los cuales son: Bahía Santa Elena, Papagayo, Punta GordaPunta Pargos y Cabo Blanco. Para cada uno de estos sitios se identificaron objetos de conservación los cuales fueron determinados por: a) grado de amenaza global (según UICN); b) irremplazabilidad y c) jurisdiccionalidad; de éstos en total se identificaron 51 objetos de conservación para todo el país (Alvarado, Herrera, Corrales, Asch, \& Paaby, 2011), dentro de los cuales están las áreas de congregación de cetáceos (SINAC, 2008). 
Para la costa del Pacífico del país se han determinado que son tres especies de cetáceos (Megaptera novaeangliae, Stenella attenuata y Tursiops truncatus) las más frecuentes de avistar (Martínez-Fernández, Montero-Cordero, \& May-Collado, 2011). Específicamente para el Pacífico norte, solo en los sitios de importancia para la conservación de Papagayo y Bahía Santa Elena se ha determinado la ocurrencia y distribución de algunos cetáceos, como ballenas jorobadas, $M$. novaeangliae, que utilizan el área con fines de crianza y reproducción (Calambokidis, Steiger, Rasmussen, Urbán, Balcomb, Ladrón de Guevara, Salinas, Jacobsen, Baker, Herman, Cerchio, \& Darling 2000; May-Collado, Gerrodette, Calambokidis, Rassmusen, \& Sereg, 2005; Martínez-Fernández et al., 2011; Rasmussen, Palacios, Calambokidis, Saborío, Dalla-Rosa, Secchi, \& Stone 2007; Rasmussen, Calambokidis \& Steiger 2012; PalaciosAlfaro, Martínez-Fernández, Sánchez-Godínez, \& Venegas, 2012). En esos mismos sitios para delfín manchado, S. attenuata, se han determinado patrones de abundancia, asociados a zonas de afloramientos costeros en el Golfo de Papagayo (May-Collado \& Forcada 2012, May-Collado \& Morales-Ramírez 2005) o a las épocas lluviosas en la zona (Martínez-Fernández et al., 2011; May-Collado \& Forcada 2012). Finalmente, el delfín nariz de botella, T. truncatus, se ha reportado para Bahía Cuajiniquil y Santa Elena, Golfo de Papagayo y parte externa de la Península de Nicoya (May-Collado et al. 2005; PalaciosAlfaro 2007; Martínez-Fernández et al. 2011).

A pesar de los estudios que se han realizado recientemente, todavía queda ausencia de información en los sitios de importancia para la conservación marina. La falta de información sobre la ecología de estas especies aumenta la vulnerabilidad de los cetáceos, ya que no se garantiza su conservación y uso sostenible para los sitios de congregación en momentos tan críticos como la reproducción, crianza o descanso. El objetivo de este estudio es identificar las áreas de congregación de cetáceos dentro y en los alrededores de los sitios de importancia para la conservación Bahía Santa Elena, Papagayo, Punta Gorda-Punta Pargos y Cabo Blanco (Pacífico norte costarricense), mediante la recopilación de información de estudios científico previos y los muestreos en los sitios identificados. El propósito es que la información básica de las áreas de congregación se convierta en insumo para la toma de decisiones en la gestión de recursos marinos de la zona.

\section{MATERIALES Y MÉTODOS}

Área de Estudio: El estudio se realizó en los siguientes sitios de importancia para la conservación marina:

Santa Elena: Sitio cercano a Cuajiniquil; colindante al Parque Nacional Santa Rosa, ubicado en $10,939957^{\circ} \mathrm{N}, 85,762263^{\circ} \mathrm{W}$. Es una zona con acantilados de roca dura con islas e islotes. Posee fondos duros infralitorales, formaciones coralinas y un área de surgencia. Las profundidades no sobrepasan los $50 \mathrm{~m}$.

Papagayo: Colinda con el Parque Nacional Santa Rosa, ubicado en $10,77504^{\circ} \mathrm{N}$, $85,744641^{\circ} \mathrm{W}$. Está influenciado por un área de afloramiento temporal de aguas subsuperficiales (McCreary et al. 1989), con intervalos batimétricos que van de 0 a 200m (SINAC, 2008).

Punta Gorda-Punta Pargos: Se encuentra entre las comunidades Playas del Coco y Junquillal; ubicado en $10,196829^{\circ} \mathrm{N}$, $85,758869^{\circ} \mathrm{W}$. Presenta acantilados de roca dura, fondos sublitorales de lodo litoclástico y bioclástico, fondos duros infralitorales y circalitorales. Posee islas e islotes, formaciones coralinas y manglares con profundidades entre los 0 a 200m (SINAC, 2008).

Cabo Blanco: Área junto a la Reserva Biológica Cabo Blanco; ubicada en $9,534221^{\circ} \mathrm{N}$, $85,010558^{\circ} \mathrm{W}$. Muestra una costa abierta de colinas con acantilados rocosos y una plataforma muy estrecha con intervalos batimétricos que van de 0 a 200m (SINAC, 2008).

\section{Toma de datos:}

\section{Identificación de sitios de congregación}

La distribución del esfuerzo de muestreo, meses y años para cada sitio se detallan en 


\section{CUADRO 1}

\begin{tabular}{|c|c|c|c|c|c|c|}
\hline Sitio & Epoca & $\begin{array}{l}\text { Horas } \\
\text { muestreo }\end{array}$ & $\begin{array}{c}\text { Días } \\
\text { muestreo }\end{array}$ & $\begin{array}{c}\text { Tamaño } \\
\text { grupal promedio }\end{array}$ & $\begin{array}{l}\text { Número } \\
\text { avistamientos }\end{array}$ & Especies \\
\hline \multirow[t]{7}{*}{ Santa Elena } & May $2005-\mathrm{Ab} 2006^{1}$ & 159 & 22 & 10 & 23 & S. attenuata \\
\hline & & & & 20 & 3 & T. truncatus \\
\hline & & & & 2 & 10 & M. novaeangliae \\
\hline & En 1999-Nov $2000^{2}$ & ND & ND & 10 & 76 & S. attenuata \\
\hline & En-Ab $2012^{3}$ & 88 & 11 & 24 & 18 & S. attenuata \\
\hline & & & & 18 & 2 & T. truncatus \\
\hline & & & & 2 & 15 & M. novaeangliae \\
\hline \multirow[t]{2}{*}{ Punta Pargos-Punta Gorda } & Feb $2012^{3}$ & 32 & 4 & 28 & 11 & S. attenuata \\
\hline & & & & 2 & 1 & T. truncatus \\
\hline \multirow[t]{5}{*}{ Cabo Blanco } & En-Ab $2012^{3}$ & 132 & 33 & 40 & 7 & S. attenuata \\
\hline & & & & 2 & 18 & M. novaeangliae \\
\hline & & & & 6 & 2 & T. truncatus \\
\hline & Nov-Dic $2007^{4}$ & 22 & 6 & 11 & 1 & S. attenuata \\
\hline & & & & 1 & 2 & M. novaeangliae \\
\hline \multirow[t]{2}{*}{ Papagayo } & Feb-Nov 2000-20012 & ND & ND & 9 & 30 & S. attenuata \\
\hline & & & & 1 & 10 & M. novaeangliae \\
\hline Total & & & & & 229 & \\
\hline
\end{tabular}

En:Enero, Feb:Febrero, Ab:Abril, May:Mayo, Nov:Noviembre, Dic:Diciembre, ND: Datos no disponibles en publicación

${ }^{1}$ Martínez et al., 2011, ${ }^{2}$ May-Collado et al., 2005, ${ }^{3}$ Presente estudio, ${ }^{4}$ Palacios \& Rodríguez

el Cuadro 1. Para la zona de Santa Elena se realizaron recorridos durante tres o cuatro días consecutivos por mes, según el trabajo de May-Collado \& Morales-Ramírez, (2005) y Martínez-Fernández et al. (2011). En la zona de Punta Gorda-Punta Pargos y Cabo Blanco basados en los métodos de estudios previos (e.g. May-Collado \& Forcada, 2005; MonteroCordero \& Lobo, 2010; Martínez-Fernández et al., 2011), se realizaron recorridos en bote siguiendo transectos de un kilómetro de ancho paralelos a la costa $(500 \mathrm{~m}$ a cada lado del transecto), a una distancia máxima de $10 \mathrm{~km}$ de distancia. De manera complementaria, se utilizaron los datos previamente publicados por May-Collado et al. (2005), Martínez-Fernández et al. (2011) y Palacios \& Rodríguez (2008) para Papagayo, Santa Elena y Cabo Blanco, repectivamente (Cuadro 1). En cada avistamiento se registró al menos la posición geográfica, especie y fecha. También se estimó el tamaño de grupo, definido como el número estimado de animales dentro de un radio de 200 metros de distancia. Una vez tomados los datos, se continuó con el recorrido.

\section{Identificación de amenazas}

Las principales amenazas identificadas en cada sitio de importancia para la conservación tomó como base lo identificado en el proceso de GRUAS II (SINAC, 2008). Tomando en cuenta que este proceso fue hace varios años, las mismas fueron re-validadas y priorizadas a través de entrevistas abiertas con funcionarios de las Áreas de Conservación Guanacaste y Tempisque (ACG y ACT) del SINAC, análisis de documentos de planificación (Planes de Manejo y Estado de la Nación) de los últimos 5 años y observaciones en campo durante el 2012 y 2013.

Análisis de los datos: Como lo sugiere Evans \& Hammond 2004, para las regiones en 
donde solo se cuenta con información básica sobre cetáceos, puede iniciarse con la determinación de la distribución y estacionalidad de las especies, basados en muestreos con avistamientos incidentales o con plataformas de oportunidad. Debido a que existieron variantes metodológicas entre sitios y en periodos de muestreo, se aplicó en este estudio un análisis espacial para determinar los sitios de congregación. El análisis toma como supuesto que la probabilidad de encuentro de cetáceos dentro de cada sitio es la misma, ya que hay homogeneidad de hábitat y los sitios son menores a 250 $\mathrm{km}^{2}$. Lo anterior toma en cuenta que los cuatro sitios de estudio no sobrepasan los $200 \mathrm{~m}$ de profundidad y se apoya en la presencia de las principales especies de cetáceos a nivel costero $(<10 \mathrm{~km})$, reportada previamente por MartínezFernández et al. (2011) en el Pacífico del país. Adicionalmente, según Rasmussen et al. (2007, 2012), las variables que definen la presencia de ballenas son profundidad $(\leq 200 \mathrm{~m})$ y temperatura (promedio anual $=28.8^{\circ} \mathrm{C}$ ), las cuales son homogéneas dentro del área del análisis según sitios priorizados por el SINAC (2008). Es así como el análisis geo estadístico de Kriging ordinario logra predecir linealmente la continuidad espacial de los grupos de cetáceos avistados en los sitios de muestreo, basado en la interpolación de los datos de avistamientos (discontinuos), para así determinar su continuidad en el espacio no muestreado adyacente, dentro del mismo hábitat homogéneo. Esta estimación se basa en la función continua, que explica el tamaño de grupo en las distintas direcciones del espacio. Por lo tanto, los valores de tamaño de grupo serán más parecidos entre más cercanos estén, pero no serán necesariamente cercanos a la media poblacional. Se utilizó el programa $\mathrm{R}_{2.15}$ para correr el modelo.

\section{RESULTADOS}

Se reporta un total de 229 avistamientos de ballenas y delfines, dentro y en los alrededores de los sitios de importancia para la conservación marinos tomados en distintos años (ver cuadro 1). Se observaron tres especies: 166 avistamientos de Stenella attenuata (72\%), 55 avistamientos de Megaptera novaeangliae (24\%) y 8 avistamientos de Tursiops truncatus (4\%).

Basados en el tamaño de grupo y posiciones geográficas, el análisis de Kriging ordinario identifica las zonas de mayor concentración para las dos especies más abundantes, delfines manchados y ballenas jorobadas (Fig. 1 y 2). Para el delfín manchado, el modelo predice tamaños más grandes para grupos de delfines en: i) alrededores de Bahía Santa Elena, ii) al sur del Parque Nacional Marino Las Baulas y iii) alrededores de Reserva Natural Cabo Blanco. (Fig. 1). Para la ballena jorobada, las áreas de mayor concentración de avistamientos grupales, incluidos los de madre y cría, son: Bahía Santa Elena y frente a Playa Tambor. Las ballenas solitarias fueron más avistadas en el Golfo de Papagayo y en las cercanías de Reserva Natural Cabo Blanco (Fig. 2).

\section{DISCUSIÓN}

Al igual que se ha reportado previamente en otras zonas costeras del Pacífico del país, los sitios dentro y en los alrededores de los sitios de importancia para la conservación presentan avistamientos de las especies más comunes (May-Collado \& Morales-Ramírez, 2005; Martínez-Fernández et al., 2011; May-Collado \& Forcada, 2012). En general los delfines manchados y las ballenas jorobadas fueron las especies con mayor número de avistamientos y el análisis resalta áreas de congregación de estas especies. Cabe resaltar que el esfuerzo de muestreo fue distinto en espacio y tiempo por lo que es recomendable en estudios futuros homogenizar el trabajo de campo en el mismo periodo de tiempo. La alta concentración de avistamientos de delfines manchados cerca de Bahía Santa Elena, Parque Nacional Marino Las Baulas y Reserva Natural Cabo Blanco, podría estar asociada a la presencia de afloramientos estacionales, los cuales proveen una fuente importante de alimento para los delfines manchados, como ha sido demostrado para otros cetáceos (Thompson, Sydeman, Santora, 


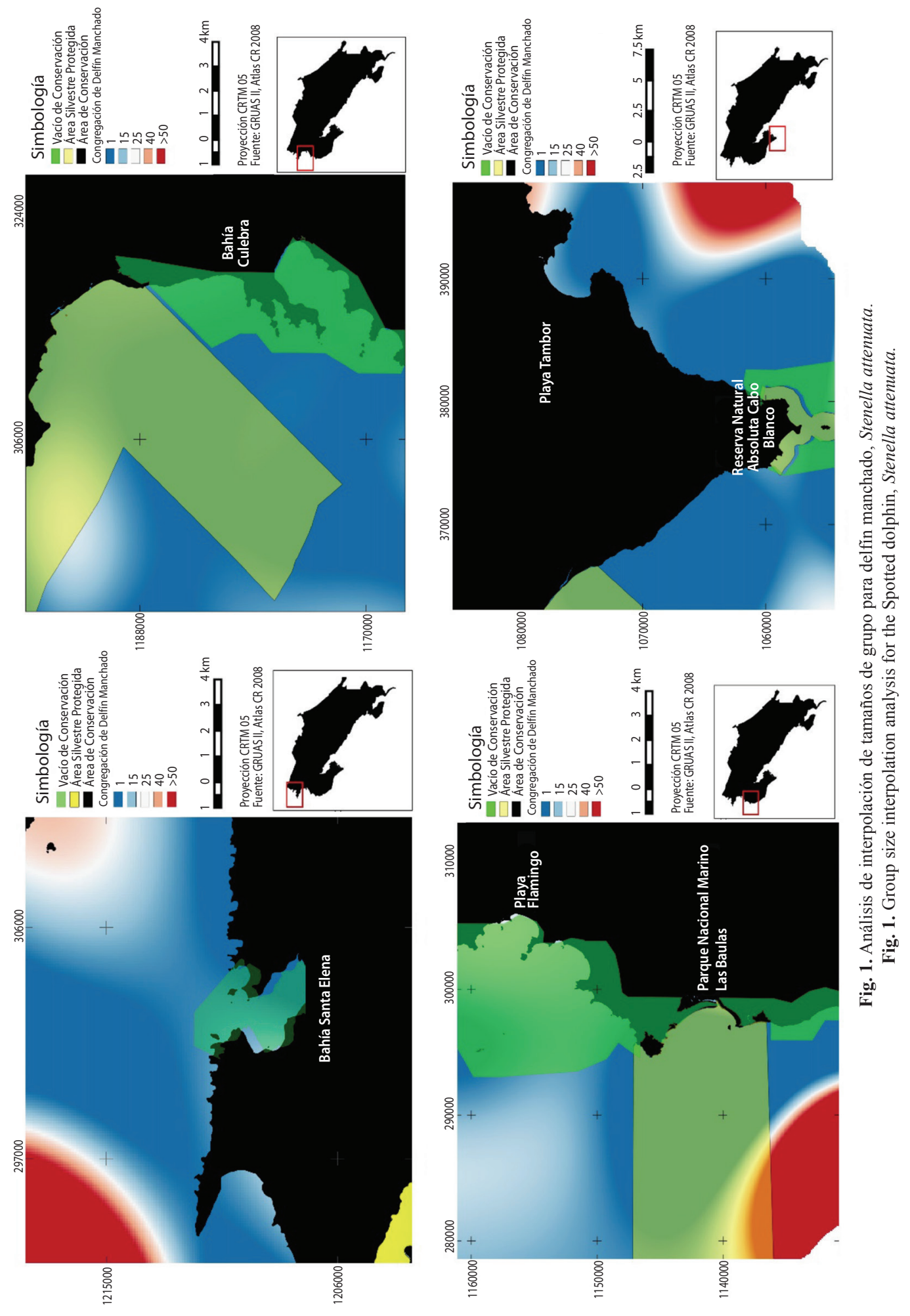



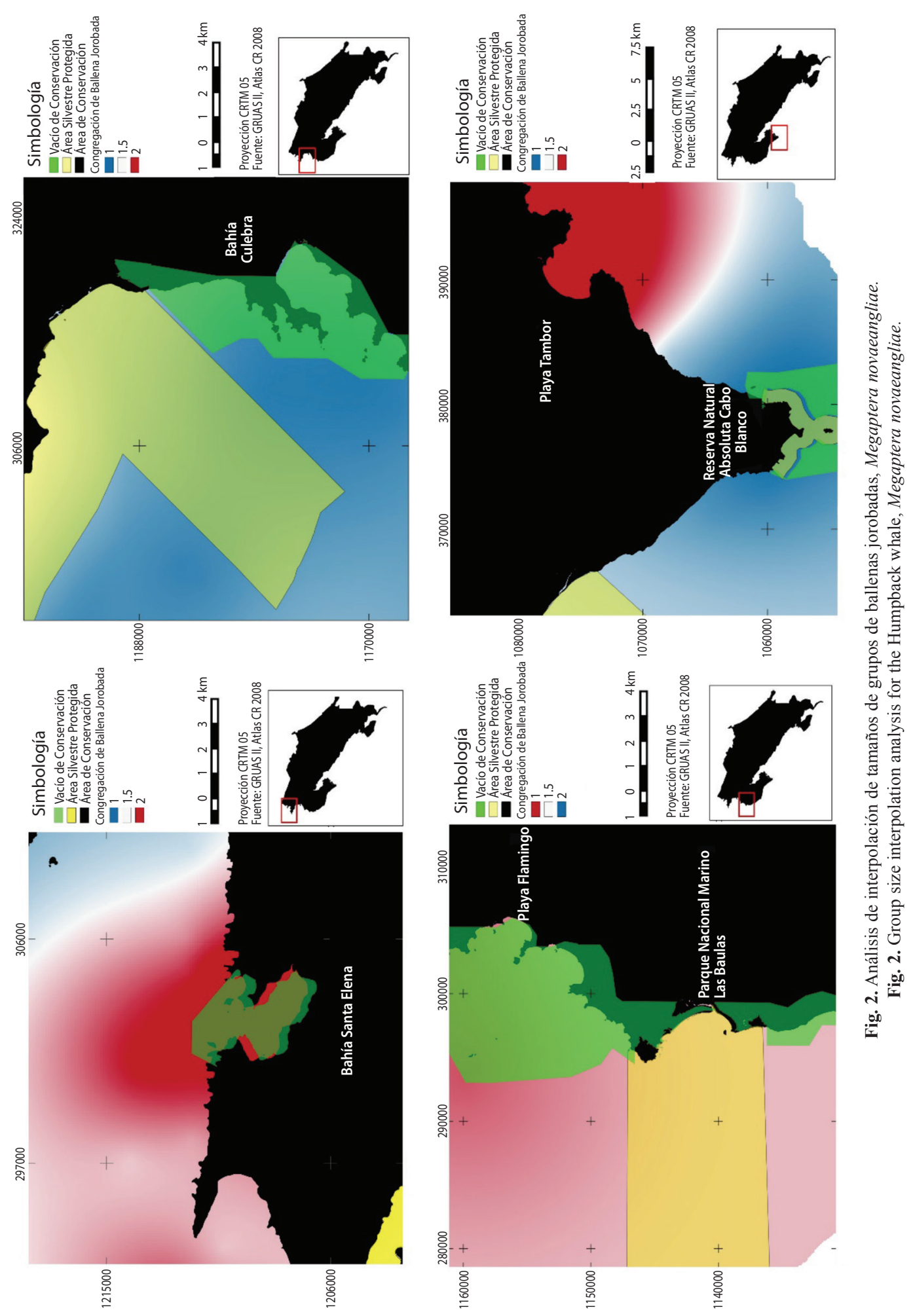
Black, Suryan, Calambokidis, \& Bograd 2012). Estudios anteriores han encontrado una asociación entre la abundancia de grupos que invierten más tiempo alimentándose en estas áreas (May-Collado \& Morales-Ramírez 2005, May-Collado y Forcada 2012).

Si bien se ha evidenciado previamente que en estas latitudes, las ballenas utilizan estas zonas para reproducción y crianza (AcevedoGutiérrez \& Smultea, 1995; Flórez-González et. al., 1998; Clapham \& Mead, 1999 Rasmussen et al., 2007, 2012.), el presente estudio brinda más detalles con respecto al uso de estos sitios, específicamente en Bahía Santa Elena y frente a Playa Tambor.

El estudio muestra que las áreas de concentración de delfines manchados y ballenas jorobadas se encuentran tanto en aguas bajo alguna categoría de manejo, como aquellas sin estas. Sin embargo, es importante notar que las congregaciones de cetáceos no parecen presentarse en zonas cercanas a las grandes comunidades costeras como Playas del Coco o Flamingo. Entre las áreas que carecen de algún tipo de manejo formal, resaltan zonas como Bahía Santa Elena, el área frente a Punta Pargos y las zonas en los alrededores de Reserva Natural Absoluta Cabo Blanco. Se recomienda en los sitios acá identificados como "sitios de congregación de cetáceos" facilitar procesos que consoliden un ordenamiento de las actividades, idealmente, definiendo una zonificación de usos entre sectores. Lo anterior facilitaría la conservación de sitios críticos de reproducción y crianza de cetáceos. Hooker et al (2011) recomiendan el mapeo de distribución de especies y sus densidades en conjunto con la caracterización de amenazas como primer paso para lograr obtener modelos predictivos de un hábitat.

Las siguientes fueron las principales amenazas identificadas en este sitio a través del estudio: i) Tráfico y turismo marino: una alta densidad de botes en el mar aumenta la vulnerabilidad de los cetáceos al ruido y a las colisiones con embarcaciones tanto con especies de ballenas como de delfines (IWC, 2008). Existe una alta actividad turística en sitios como El Coco y Tamarindo (Fonseca, 2012), localidades cercanas al sitio Punta Gorda-Punta Pargos. Adicionalmente, el Golfo de Papagayo, es la región de mayor desarrollo turístico en el país (Cajiao, 2012). Estos factores facilitan el desarrollo de actividades turísticas marinas, como es el caso del avistamiento de cetáceos. Existen claros registros de cómo esta actividad de avistamiento puede incurrir en alterar comportamientos críticos de delfines y ballenas (Montero-Cordero \& Lobo, 2010) e incluso llegar a abandonar un sitio anteriormente utilizado (Lusseau \& Bejder, 2007), ii) Pesca semiindustrial: Para la pesca de arrastre los efectos negativos de este tipo de arte están demostrados para los cetáceos, tanto de manera directa, por pesca incidental (Lewison, Crowder, Read, \& Freeman, 2004; Mannocci, Dabin, AugeraudVéron, Dupuy, Barbraud, \& Ridoux, 2012), como indirecta, por degradación de hábitat (Chilvers, \& Corkeron, 2001; Fock, 2011), iii) Pesca pequeña escala: la pesca incidental y los enmallamientos de ballenas y delfines son un problema a nivel mundial. Se estima que más de 650000 mamíferos marinos mueren al año debido al enredo en redes de pesca, pesca fantasma o pesca incidental (Read, Drinker, \& Northridge, 2006; Butterworth, Clegg, \& Bass, 2012) y en estas zonas son frecuentes las comunidades pesqueras y iv) Desarrollo urbanístico: Se ha demostrado que la contaminación por aguas residuales (TT Argos, 2010a,b) y degradación de hábitats marinos (e.g. Fernández \& Cortés, 2005) tienen impactos directos e indirectos sobre cetáceos costeros (Jefferson, Hung \& Würsig, 2009)

Con base en lo anterior, se propone que para mejorar la gestión de los cetáceos como elementos focales de manejo en estos sitios de importancia para la conservación marinos, se prioricen las siguientes acciones: i) determinar las rutas reales de tráfico marino en las zonas para que sean insumo para la Ley de Navegación Acuática, de esta manera se puede integrar a las labores de ordenamiento y control del transporte marítimo (Delgado \& Zeledón, 2013), establecer una clara zonificación para los sitios de observación y velar por el cumplimiento del decreto Ejecutivo 32495 (2005) 
y de manera complementaria aplicar códigos voluntarios de buenas prácticas para el turismo marino, con base en resultados registrados para otras comunidades costeras (e.g. MolinaBustamante et al., 2011), ii) cumplir con lo dispuesto en el Voto de la Sala Constitucional (2013) en donde, con base en pruebas técnicas del impacto a nivel regional, se prohíbe otorgar, reactivar o renovar permisos para la pesca de camarón de arrastre, iii) tomando en cuenta que los pescadores artesanales tienen mucho que aportar en los esfuerzos nacionales orientados a la conservación de la biodiversidad marina (Solís-Rivera, 2005; Quirós, Pesquero, Gómez, Solano, Antman, Nuñez, Marquez, Pérez, \& Jiménez, 2012), se recomienda hacer partícipes a los grupos organizados del sector de pequeña escala a la hora de planificar e implementar estrategias de conservación dentro y fuera de áreas marinas protegidas y iv) actualizar e implementar los planes reguladores cantonales y costeros a través de la coordinación de acciones entre Municipalidades, Ministerio de Ambiente y Ministerio de Salud.

En conclusión, a pesar de que se han identificado sitios de congregación de cetáceos tanto en áreas protegidas como no protegidas del Pacífico norte, resaltan las zonas que al momento no poseen ningún tipo de manejo, como Bahía Santa Elena, Bahía Culebra, área frente a Punta Pargos y alrededores de la Reserva Natural Cabo Blanco. Si bien no puede garantizarse la salud de un sitio con base en la existencia de una categoría de manejo o con la protección de una especie, una potencial creación de áreas protegidas con usos directos e indirectos permitidos, sí garantizarían el establecimiento de una zonificación de actividades en un área particular. Tomando en cuenta que la situación actual costarricense no refleja una política clara para ordenar de forma integral la situación de la zona marítimo terrestre, se considera oportuno impulsar iniciativas de gestión de recursos marinos a través de la consolidación de áreas marinas protegidas existentes o creación de nuevas áreas con categoría participativas. Lo anterior facilitaría la gestión integrada para la conservación de cetáceos de hábitos costeros en el Pacífico Norte de Costa Rica.

\section{AGRADECIMIENTOS}

Agradecemos a la Agencia Alemana de Cooperación GIZ a través del Proyecto BIOMARC-BMU por el apoyo económico. También al Sistema Nacional de Áreas de Conservación, a los equipos del Área de Conservación Guanacaste, Área de Conservación Tempisque, a la Asociación Costa Rica por Siempre, al Proyecto Consolidación de Áreas Marinas Protegidas y a la Vicerrectoría de Investigación de la Universidad de Costa Rica a través del proyecto 808 -B2-400 del CIMAR. Se agradece por la colaboración en el muestreo a: Claudia Matzdorf, Luis Schultz (Refugio Nacional de Vida Silvestre Curú), Anibal Lara y Minor Lara (Centro de Buceo Cuajiniquil), Rubén Venegas y por el uso de datos a: Laura May-Collado, Tim Gerrodette, John Calambokidis, Kristin Rasmussen, Irena Sereg y Gerardo Palacios.

\section{RESUMEN}

La información disponible sobre la distribución de los cetáceos para el Pacífico Norte de Costa Rica actualmente carece de análisis que faciliten la toma de decisiones con el fin de tomar medidas políticas o de manejo para la conservación de los cetáceos costeros. El objetivo de este estudio es identificar áreas de importancia para los cetáceos en el Pacífico Norte, especialmente fuera de la de las áreas protegidas existentes. Se analizaron datos publicados anteriormente sobre avistamientos de cetáceos entre 2004 y 2008, y se complementó esta información con esfuerzo de muestreo durante la estación seca del 2012. Se utilizó un análisis geoestadístico de Kriging ordinario para predecir la continuidad espacial de avistamientos de cetáceos dentro de cuatro áreas de estudio. Con base en este análisis, las áreas de congregación más importantes para los delfines son: Bahía Santa Elena, Parque Nacional Marino Las Baulas and Reserva Natural Cabo Blanco, debido a las altas densidades de grupos de delfines en actividades de alimentación. Las áreas de congregación relevantes para las ballenas jorobadas son: Bahía Santa Elena, Playa Tambor, Golfo de Papagayo and Reserva Natural Cabo Blanco. El estudio identifica las áreas de altas concentraciones de cetáceos en el Pacífico Norte, tanto dentro como fuera de las zonas protegidas. Nuestros resultados indican que algunas de las áreas no protegidas deben ser priorizadas con 
respecto a la priorización de estrategias de conservación, tales como: Península de Santa Elena, Bahía Culebra, Santa Rosa-Islas Murciélagos, y los alrededores de Punta Pargos y Cabo Blanco. Con base en una primera identificación de las amenazas marinas, se proponen medidas de gestión y políticas para garantizar la conservación de estos sitios de congregación de cetáceos.

Palabras claves: delfin machado, ballena jorobada, áreas marinas protegidas, manejo, sitios de importancia para la conservación.

\section{REFERENCIAS}

Acevedo-Gutiérrez, A. \& Smultea, M. A. (1995). First records of humpback whales including calves at Golfo Dulce and Isla del Coco, Costa Rica, suggesting geographical overlap of northern and southern hemisphere populations. Marine Mammal Science, 11(4), 554-560.

Alvarado, J. J., Herrera, B., Corrales, L., Asch, J., \& Paaby, P. (2011). Identificación de las prioridades de conservación de la biodiversidad marina y costera en Costa Rica. Revista de Biología Tropical, 59(2), 829-842.

Butterworth, A., Clegg, I., \& Bass, C. (2012). Untangled Marine Debris: A Global Picture of the Impact on Animal Welfare and of Animal-focused Solutions. London, Reino Unido: WSPA International.

Cajiao, M. V. (2012). Aspectos legales del Polo Turístico Golfo de Papagayo, Guanacaste, Costa Rica: régimen especial. Revista de Biología Tropical, 60 (Suplemento 2),),), 225-230.

Calambokidis, J., Steiger, G. H., Rasmussen, K., Urbán R., J., Balcomb, K. C., Ladrón de Guevara P., P., Salinas Z., M., Jacobsen, J. K., Baker, C. S., Herman, L. M., Cerchio, S., \& Darling, J. D. (2000). Migratory destinations of humpback whales that feed off California, Oregon and Washington. Marine Ecology Progress Series, 192, 295-304.

Chilvers, B. L., \& Corkeron, P. J. (2001). Trawling and bottlenose dolphins' social structure. Proceedings of the Royal Society of London. Series B: Biological Sciences, 268(1479), 1901-1905.

Clapham, P. J., \& Mead, J. G. (1999). Megaptera novaeangliae. Mammalian Species, (604), 1-9.

Decreto Ejecutivo No 32495-MINAE-MOPT-MSP-MAG. (2005). Reglamento para la Operación de Actividades Relacionadas con Cetáceos en Costa Rica... La Gaceta, Número 145 del 28 de julio del 2005, 6 p.

Delgado, K., \& Zeledón, N. (2013). Proyecto de Ley de Navegación, Expediente 18.512 Oficio ST.090-2013. San José, Costa Rica: Departamentos de Servicios Técnicos, Asamblea Legislativa de la República de Costa Rica. 89 p.
Evans, P. G., \& Hammond, P. S. (2004). Monitoring cetaceans in European waters. Mammal review, 34(1-2), 131-156.

Fernández, C., \& Cortés, J. (2005). Caulerpa sertularioides, a green alga spreading aggressively over coral reef communities in Culebra Bay, North Pacific of Costa Rica. Coral Reefs, 24(1), 10-10.

Flórez-González, L., Capella, A.J., Haase, B., Bravo, G.A., Félix, F.,\& Gerrodette, T. (1998). Changes in winter destinations and the northernmost record of southeastern Pacific humpback whales. Marine Mammal Science, 14(1), L89-196.

Fock, H. (2011). Integrating multiple pressures at different spatial and temporal scales: a concept for relative ecological risk assessment in the European marine environment. Human and Ecological Risk Assessment, 17(1), 187-211.

Fonseca, A. (2012). Gestión marino-costera. Decimoctavo Informe Estado de la Nación en Desarrollo Humano Sostenible. Informe final. San José, Costa Rica: Programa Estado de la Nación,,, 82 p.

Hooker, S. K., Cañadas, A., Hyrenbach, K. D., Corrigan, C., Polovina, J. J., \& Reeves, R. R. (2011). Making protected area networks effective for marine top predators. Endangered Species Research, 13, 203-218.

IWC-International Whaling Commission. (2008). Agenda Item 15: Report of the Conservation Committee, at 1-4, IWC Doc. IWC/60/Rep 5. 60th Annual Meeting of the IWC, Santiago, Chile, June 23-27, 2008,

Jefferson, T. A., Hung, S. K., \& Würsig, B. (2009). Protecting small cetaceans from coastal development: Impact assessment and mitigation experience in Hong Kong. Marine Policy, 33(2), 305-311.

Lewison, R. L., Crowder, L. B., Read, A. J., \& Freeman, S. A. (2004). Understanding impacts of fisheries bycatch on marine megafauna. Trends in Ecology \& Evolution, 19(11), 598-604.

Lusseau, D., \& Bejder, L. (2007). The long-term consequences of short-term responses to disturbance experiences from whalewatching impact assessment. International Journal of Comparative Psychology, 20(2), 228-236...

Mannocci, L., Dabin, W., Augeraud-Véron, E., Dupuy, J. F., Barbraud, C., \& Ridoux, V. (2012). Assessing the impact of bycatch on dolphin populations: the case of the common dolphin in the eastern North Atlantic. PloS ONEONEONE, 7(2), e32615.

Martínez-Fernández, D., Montero-Cordero, A. \& MayCollado, L. J. (2011). Cetáceos de las aguas costeras del Pacífico norte y sur de Costa Rica. Revista de Biología. Tropical, 59, 283-290.

May-Collado L, Gerrodette T, Calambokidis J, Rasmussen K, Sereg I (2005). Patterns of cetacean sighting distribution in the Pacific exclusive economic zone of 
Costa Rica based on data collected from 1979-2001. Revista de Biología. Tropical 53 (1-2), 249-263

May-Collado, L. \& Morales Ramírez, A. R. (2005). Presencia y patrones de comportamiento del delfín manchado costero, Stenella attenuata graffmani (Cetacea: Delphinidae) en el Golfo de Papagayo, Costa Rica. Revista de Biología. Tropical, 53, 265-276.

May-Collado, L. J., \& Forcada J. (2012) Small-scale estimation of relative abundance for the coastal spotted dolphins (Stenella attenuata) in Costa Rica: the effect of habitat and seasonality. Revista de Biología. Tropical, 60 (Suplemento 2), 133-142.

McCreary, J. P., Lee, H. S., \& Enfield, D. B. (1989). The response of the coastal ocean to strong offshore winds: With application to circulations in the Gulfs of Tehuantepec and Papagayo. Journal of Marine Research, 47(1), 81-109.

Molina-Bustamante, C., Martínez-Fernández, D., \& Montero-Cordero, A. 2011. Implementation of a Best Practices ecolabelling system among marine tour operators. 2nd International Marine Conservation Congress. 14-18 Mayo, 2011. Victoria, Canada. Presentación Oral.

Montero-Cordero, A. \& Lobo, J. (2010). Effect of tourist vessels on the behaviour of the pantropical spotted dolphin in Drake Bay and Cano Island, Costa Rica. Journal of Cetacean Research and Management 11(3): 285-291.

Palacios, G. \& Rodriguez, J. (2008). Justificación Técnica para la Ampliación del Área Marina de la Reserva Natural Absoluta Cabo Blanco (RNACB). Guanacaste, Costa Rica: Área Conservación Tempisque, 52 p.

Palacios-Alfaro, D., Martínez-Fernández, D., SánchezGodínez, C. \& Venegas, R. (2012). Distribution and behavior of humpback whale (Megaptera novaeangliae Borowski, 1781) (Breeding Stock G), in southern Pacific of Costa Rica. Panama: Paper SC/64/SH16 IWC Scientific Committee, July 2012.

Palacios-Alfaro, J. D. (2007). Presencia y comportamiento de dos especies de delfines en el Pacífico central de Costa Rica. Tesis de Graduación. Heredia, Costa Rica: Universidad Nacional, $70 \mathrm{p}$

Quirós, W., Pesquero, M., Gómez, F., Solano, M., Antman, P.,.,., Nuñez, J., Marquez, A., Pérez, J., \&\&\& Jiménez, A. (2012). Caracterización de las pesca artesanal de pequeña escala dentro y en las cercanías del Refugio Nacional de Vida Silvestre Ostional, Guanacaste, Costa Rica. San José, Costa Rica: Bioma-ISV, 60 p.

Rasmussen, K., Calambokidis, J., \& Steiger, G. H. (2012). Distribution and migratory destinations of humpback whales off the Pacific coast of Central America during the boreal winters of 1996-2003. Marine Mammal Science, 28(3), E267-E279.
Rasmussen, K., Palacios, D. M., Calambokidis, J., Saborío, M. T., Dalla-Rosa, L.,Secchi, E. R., \& Stone, G. S. (2007). Southern Hemisphere humpback whales wintering off Central America: insights from water temperature into the longest mammalian migration. Biology Letters, 3(3), 302-305.

Read, A. J., Drinker, P., \& Northridge, S. (2006). Bycatch of marine mammals in US and global fisheries. Conservation Biology, 20(1), 163-169.

Sala Constitucional de la Corte Suprema de Justicia (2013). Expediente: 12-010016-007-CO. Res. No 2013010540. San José, Costa Rica: Corte Suprema de Justicia. 140p.

SINAC-Sistema Nacional de Áreas de Conservación. (2008). GRUAS II: Propuesta de Ordenamiento Territorial para la conservación de la biodiversidad de Costa Rica. Volumen 3: Análisis de vacíos en la representatividad e integridad de la biodiversidad marina y costera. San José, Costa Rica: Asociación Conservación de la Naturaleza, 60 pp.

Solís-Rivera, V. (2005). El mar y su gente: la conservación de los recursos marino costeros. VII Congreso Nacional de Ciencias. Heredia, Costa Rica: Fundación CIENTEC.

Thompson, S. A., Sydeman, W. J., Santora, J. A., Black, B. A., Suryan, R. M., Calambokidis, J., ... \& Bograd, S. J. (2012). Linking predators to seasonality of upwelling: using food web indicators and path analysis to infer trophic connections. Progress in Oceanography, 101(1), 106-120.

TT Argos. (2010a. Dinámica territorial del desarrollo turístico costero: Unidad Turística Guanacaste Norte. Caracterización de la Unidad Turística Guanacaste Norte, incluyendo aspectos históricos, físicos, ambientales y socioeconómicos, así como su dinámica turística. Informe final, capítulo II. In El Impacto del Desarrollo Asociado al Turismo en la Costa del Pacífico de Costa Rica. Center for Responsible Travel, CREST, Stanford University and Washington, DC. 55 p.

TT Argos. (2010b). Dinámica territorial del desarrollo turístico costero: Unidad Turística Guanacaste Sur. Caracterización de la Unidad Turística Guanacaste Norte, incluyendo aspectos históricos, físicos, ambientales y socioeconómicos, así como su dinámica turística. Informe final, capítulo III. In El Impacto del Desarrollo Asociado al Turismo en la Costa del Pacífico de Costa Rica. Center for Responsible Travel, CREST, Stanford University and Washington, DC. 58 p.

Wehrtmann, I. S., \& Cortés, J. (Eds.). (2009). Marine Biodiversity of Costa Rica, Central America. Berlín, Alemania:Springer. 